\title{
A Framework of Social Media Messages for Crisis and Risk Communication: A Study of the Covid-19 Pandemic
}

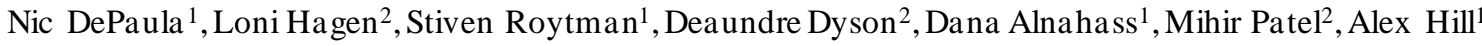 \\ Wayne State University ${ }^{1}$, University of South Florida ${ }^{2}$ \\ ndepaula@wayne.edu, lonihagen@usf.edu,stiven.roytman@wayne.edu, \\ ddyson@usf.edu,hh4056@wayne.edu,mkpatel@usf.edu, alexbhill@wayne.edu
}

\begin{abstract}
Social media are important channels for crisis and risk communication by government agencies. However, existing frameworks for studying these messages use loose and inconsistent terminology, making it difficult to build on this research and understand how message features impact message diffusion. In this study, we provide a framework based on textual and media dimensions of messages for improved analysis of social media crisis and risk communication. We apply the framework to a sample of Twitter posts from United States local, state and federal public health agencies during a year of the Covid-19 pandemic. Results show reasonable reliability levels for coding of message features; differences and similarities of messages across local, state and federal public health agencies; and significant associations between message features and message diffusion. The study contributes to research on crisis and risk messages, and our understanding of the impacts of message features on message diffusion.
\end{abstract}

\section{Introduction}

In periods of crisis, reliable information and communication by government agencies can mitigate harmful impacts and are an essential part of crisis management $[1,2]$. On social media in particular, public health messages can be retransmitted across networks by the public itself, widening messa ge reach $[3,4,5]$. While social media are notable notification systems for extreme events [3], they have also been institutionalized as part of government [6], and are employed by various types of local, state and federal a gencies in crisis and risk communication [3,4,7].

Despite the importance of a social media presence, there is no unifying framework for analysis of government social media messages during crises. Existing frameworks seem to use ad-hoc $[8,9]$, or a purely based on a lexical approach $[10,11]$. Studies often do not distinguish intention of messages (e.g. "inform", "increase resilience" [10, 12, 13]) from policies (e.g. "closures/openings" [10]), or everything is called a "topic" [8]. Sometimes the categories for message analysis are themselves metaphorical (e.g. "fighting rumours" [13]) or are vague and difficult to validate (e.g. "open and transpa rent messages"[14]).

Previous studies have distinct purposes and have contributed to our understanding of crisis and risk communication messages on social media. However, the "categories", [9, 15], "strategies" [16], "frames" [9] or "features" [11] of crisis and risk messages could benefit from a more linguistically informed framework; one that distinguishes speech acts (e.g. message purpose or intention) from topics (e.g. risk information), while also including other relevant dimensions of social media messages such as speaker, audience and types of images - which are important but not previously systematically explored [1, 17].

Such a framework could help integrate the various message features from the literature and help formalize analysis in this domain. Also, integration can help us better understand the impact of message features on message diffusion. Existing studies have several findings about the impact of message features on rates of message diffusion (i.e. public sharing) in crisis situations $[8,9,10,16]$, but given inconsistent terminology it is difficult to build upon them.

To contribute to the literature, we thus provide a framework of government social media messages for crisis and risk communication based on textual and media analysis [17, 18, 19]. The framework integrates categories from the literature, adds additional ones, and focus on the syntax and semantics of the texts. We also asked the following research questions (RQs):

$\mathrm{RQ1}$. How reliable are the framework features for analyzing crisis-related social media messages?

RQ2. How are message features employed across levels of government agencies throughout a crisis?

RQ3. How are specific message features associated with message diffusion rates?

We addressed these questions via a case analysis of a sample of Covid-19 related "tweets" (also referred to as posts or messages) from 85 local, state and federal 
United States (US) public health agencies main Twitter accounts, covering the year of 2020, across multiple waves of the pandemic.

We found the features of the proposed framework to have mostly moderate and strong inter-rater reliability measures. We also observed interesting patterns in the use of Twitter and message features across agency levels, some of it following waves of the pandemic. We also observed significant relationships between certain message features and their rates of diffusion.

In the following sections, we present a literature review, methods, and findings, followed by our discussion in light of existing theories about message design and the goals of public health and government agencies. We conclude with study limitations and directions for future research.

\section{Literature Review}

\subsection{Crisis and risk communication on social media by government agencies}

Social media (SM) have been widely adopted by government agencies to communicate with the public in crises $[3,4,8]$. Communication is important in these situations to help the public make informed decisions, and reduce overall public harm $[1,20,21]$. In the US most adults use social media [22], and they are widely adopted by emergency management [3] and public health agencies [8]. Given wide adoption of SM in society and government, it is useful to understand the expectations of the public and agencies in these environments. Better understanding of the textual and media elements could also lead to better government communication strategies, and potential differences across local, state and federal agencies [7]. Since SM allow for public sharing of messages $[4,5,10]$, which increases message reach, understanding how features play a role in message diffusion can help instruct guidelines for better message design.

\subsection{Features of government crisis and risk communication messages}

Crisis and risk communication (CRC) messa ges on SM have been largely studied and have various relevant features. For example, a popular "genre analysis" proposed 5 "top-level genres" (broadcast information, broadcast warning, encourage behavior, appeal for information, fighting rumours) to categorize flood [13] and earthquake emergency communications [12]. Others have examined "condolences" and "encouragement" [4] messages, while recent studies examined "resilience" and "susceptibility" content, a mong others [10,11].

While these approaches provide useful and unique analyses, "message content" is often defined in a nebulous way. For example, studies often employ "information" as a category, when any part of any a message can be considered information $[3,4,12]$. The notion of a "warning" is a lso difficult to observe from text unless explicitly stated, as any message about a crisis or risk can be a warning.

In this section, we provide a framework for analysis of crisis and risk social media messages based on linguistic theories of textual analysis [18, 19] and image use in risk messages [17]. We incorporate categories/features from the literature, and add some not previously explored, including speakers, audience and image types, We also discuss previous results on associations between message feature and diffusion (i.e. sharing; retransmission).

The literature for this study was identified via keyword search (i.e., "social media", "message", "crisis/risk communication", "public health" and "crisis management") from, Web of Science, Scopus and Google Scholar. From an iterative review of the various themes from the literature, we identified seven broad textual dimensions and one media dimension to construct the framework. These are: speech function, topic, threat focus, type of resource, audience, speaker, rhetorical tactic and media. Each of these dimensions includes more granular message features. The framework is not exhaustive, and can be expanded. It is devised with tweets and short Facebook posts in mind. A summary of the framework is provided in Table 1 and discussed below.

Speech function. Speech functions, also called speech acts, are the distinct types of social functions that can be observed from text [18]. For example, a statement such as: "an emergency has been declared" (a representative) has the function of informing or representing something; the statement "you must evacuate the area" (a directive) has the function to command or direct an action. The first reflects an existing phenomenon; the latter attempts to bring a phenomenon into being by directing others to do it. Speech act theory is a field of research with nuanced and competing models, but this framework identifies basic speech functions that are well recognized [18, 23]. These have been previously referred to as "sentence style" of messages in CRC [3], but speech functions are more than simply "style". The first speech function is the representative, also known as assertive, and associated with the declarative form [18]. This speech function is relevant as it is associated with information provision. It describes, explains or 
Table 1. A framework of social media message features for analysis of crisis and risk communication

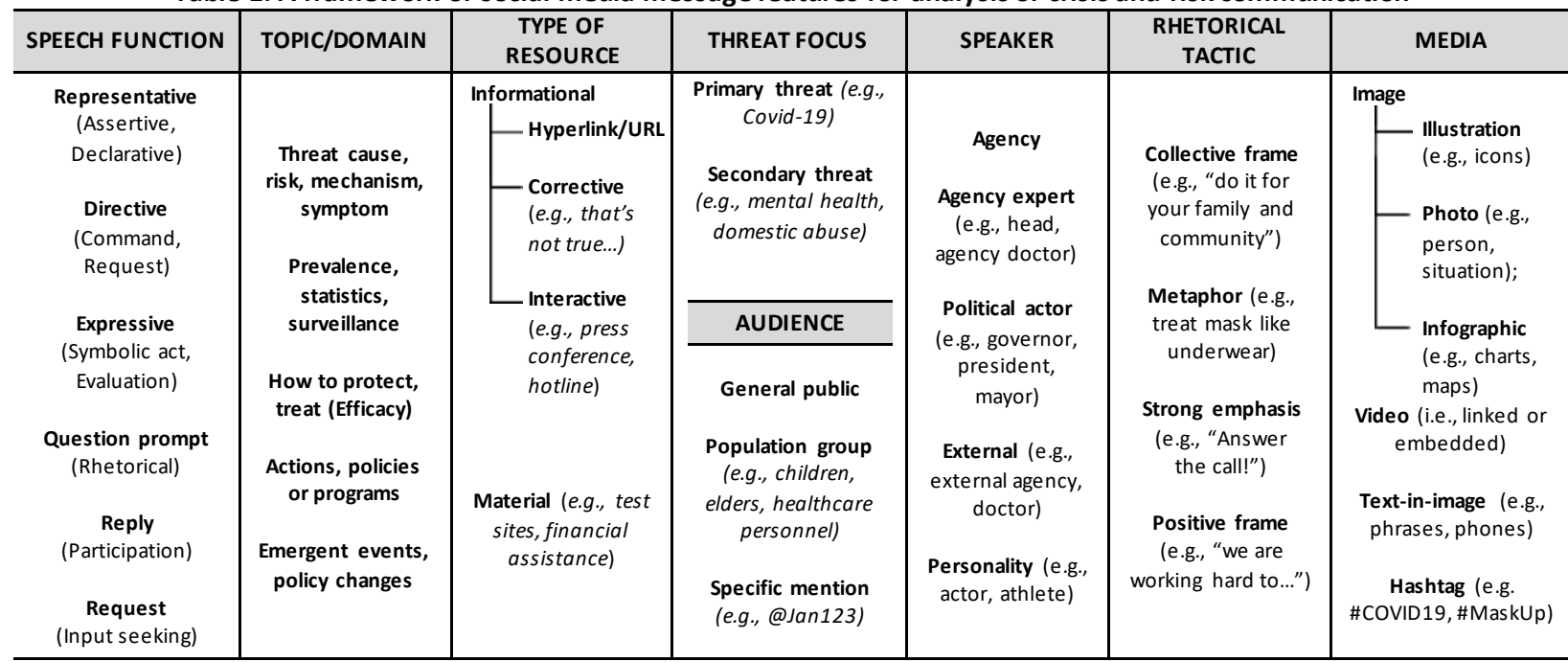

justifies phenomena [19]. In a study examining the role of these types of statements on message diffusion, it was found that the presence of a decla rative sentence had a positive but not significant association with diffusion on Twitter in a hurricane scenario [3].

Directives, also referred to as commands are statements that indicate what a person must or should do, usually in imperative tense (e.g. "Wear a mark") Searle also considered some requests as directives, since they attempt to draw a listener response [24]. In this framework, statements such as "you must" or "you should", or statements in the imperative form such as "answer the call" are considered directives. Messages with imperatives were associated with higher levels of diffusion in multiple emergency scenarios [3].

Expressives are statements that express an attitude or sentiment of the speaker. They may appear as representatives, such as "we're sad to say" or as phatic expressions such as "thanks". In the framework, we considered symbolic language such as "Be a hero!" as expressives [25]. It is important to show empathy in crisis communication [1], and others have discussed the use of "emotion-evaluative" [3], "resilience" [11] and "reassurance" message features in related contexts [9]. The presence of "emotion-evaluative" content has been found associated with higher message diffusion in multiple CRC scenarios [3], while "resilience" keywords had a more mixed but positive relationship in the context of Covid-19 [11].

A reply to a specific citizen question or comment is considered here as a distinct speech function since it is the provision of directly and specifically requested information. A reply may not be genuine or valuable, but it is a reflection of participatory government [6], potentially leading to trust and credibility.

A request is a distinct speech act because it creates an open chance for engagement with the speaker. In some cases a directive may seem like a request [24], e.g.: "Get vaccinated soon". However, requests here are statements that seek some kind of citizen input, including an answer to a question; or material assistance from the public, such as volunteering, policy participation or donations.

Previous studies have examined the role of "question marks" and "interrogative sentences" in diffusion rates, but results a re not consistent [3, 11]. Nevertheless, based on initial observations, we noted a type of rhetorical question that was prevalent in the messages, employed to identify a topic or relevant audience (e.g., "Did you know that..."). We referred to these statements as question prompts and included them as a speech function since no other speech function seemed appropriate for these clauses.

Topic/Domain. The topic of the message refers to the contexts ordomains of phenomena reflected in the text [19]. There are many specific topics or domains that could be relevant during CRC. Here we propose five general topics/domains that are largely mutually exclusive and align with categories in the literature.

Descriptions of the cause, risk, mechanism and/or impacts may seem like many topics but it reflects scientific and causal information about the threat. This is close to what others have called "symptoms", "disease mechanisms" [9], "risk and crisis information" [26], and "susceptibility" [11]. In other studies, "susceptibility" keywords had mixed correlation with message diffusion, but "symptoms" and "technical information" keywords were positively associated with message diffusion $[10,11]$.

A second feature in the model refers to information about the prevalence and statistics of the spread of the threat, which may include sophisticated surveillance information about cases, test results, etc. This is a 
relevant category as it provides reliable and generalized information about the threat prevalence that may be difficult for the public to obtain otherwise. As recently examined, this feature was particularly effective in message diffusion [11].

How to protect or treat was conceived as a distinct message feature that refers to what has been discussed as efficacy [27]. This type of information mentions how the public can protect from or treat the threat, and are important because they offer practical content. These statements may be similar to those on causes or symptoms, but more clearly focus on action to protect from risk. Messages with efficacy features were associated with message diffusion in public health [27], and emergency management a gencies [11].

Another message feature is: actions, policies or programs, which is similar to what others have called "official action" [27], "official responses" [11] and "operations" [8] most of which had weak but positive associations with message diffusion. Either way, government agencies are likely to want to show their engagement and positive actions during crises [21]. Moreover, citizens may want to know what the current actions, policies and programs a vailable are.

We conceived of a separate emergent events/policy changes to refer to messages that are more timely during an ongoing crisis. During a long-term pandemic, some messages may be more timely than others. In related studies this has been narrowly discussed as "closures/openings" [8] - found to have mixed results with message diffusion $[3,11]$ ).

Threat focus. Threat focus is the threat or risk at issue referenced in the message, of which there will be a primary threat (e.g. Covid-19). However, messages may also be about a secondary threat that arises from or are related to the primary threat [11]. In the context of the Covid-19 pandemic these have included mental health and child abuse [28, 29]. In a recent study, tweets identifying "secondary impacts" were positively associated with diffusion [11].

Type of resource. A message itself is an informational resource. Messages may also include other informational resources: hyperlinks/URLs to more information; and references to interactive resources, such as hotlines, live videos or press conferences. Messages may also correct existing mis/information or rumors that can easily spread on social media [30].

Previous research examining the role of URLs and "corrections" in CRC messages found that URLs are associated with lower message diffusion, whereas corrections had mixed results $[3,11,16]$. These same studies defined interactive resources as "information" and "information sharing" (regrettably), which had a negative association with diffusion.

We also observed that messages may refer to material resources that are available, such as tests, vaccines, financial assistance or others, which may be more valuable than informational resources alone. We thus included this as a separate feature of framework.

Audience and speaker. In CRC it is important to identify the audience of a message $[1,2]$. Since the public is diverse, and distinct information, threats or resources may target specific groups, it is important to understand the publics toward which messages are directed. We assume that if a message is posted on Twitter, it is for the general public. However, messages may indicate a population group (e.g., elders, youth, individuals with diabetes) or mention a person toward which the message is directed.

Population group as defined here includes some of what has been referred to as "susceptibility" (which we conceive as risk information), and it was found to be positively but weakly associated with message diffusion in a recent Covid-19 study [11].

Another major message dimension refers to the speaker. If a message is posted by an agency, the agency is the main speaker. But a message may quote other speakers, or identify another subject of the action. Research in risk message design have noted the importance of "celebrity-based appeals" [31], and agency messages can often figure politicians [32]. We also identify agency expert/staff, and external agent as potential speakers. It appears research has not tested how distinct speakers may improve message diffusion.

Rhetorical tactic. Rhetoric is the art of discourse, and there are several relevant tactics that can be employed in crisis and risk situations [33]. Here we describe four. The first is collective frame, which is the use of collective pronouns and references to friends, family and community, as a rationale for action. Public emergencies are inherently collective problems, and any a message may emphasize its collective nature. Collective frames have been discussed as "collective efficacy", and found to be positively, although weakly, associated with message diffusion [11].

Other rhetorical tactics are metaphors, strong emphasis, and positive frame. Since simplifying language is important for understanding risks [1], metaphors may be important in the context of clarifying scientific information. Positive frame is relevant given the role of strategic self-presentation by government agencies in social media [34]. Strong emphasis refers to the use of exclamation or capitalized letters in text. This last variable has had mixed effects on message diffusion $[3,11]$. 
Media. A social media post is its own unique medium, and social media messages may include additional media. On Twitter and Facebook, in addition to text, this usually includes: an image, a video, a hyperlink and/or a hashtag [35]. We also include a separate feature for identifying additional text in image. Recently, textual content on images of Covid-19 related messages were positively associated with diffusion, more so than the same text feature outside of the image [11]. The study of [11] found that the inclusion of an image, URL or hashtag were negatively associated with message diffusion, but others have generally found the opposite concerning the impact of images on message diffusion [10,27].

Pictures and images are important pieces of content in risk communication [17], but have not been examined in detail in previous studies of crisis and risk communication. Even in a recent study of Instagram content [26], an entirely image-based social media platform, the types of images themselves were not explored. Pictures and images can help in persuasion, comprehension and recall of messages [17]. In this study, we propose at least three types of images to consider: photographs (e.g., of people, situations); illustrations (e.g., of things or processes); and infographics (e.g., charts, maps, demonstrations).

\section{Methods}

To address our research questions, this study conducted manual coding of sampled tweets based on the framework. The annotation results were validated by calculating inter-rater reliability. We also provided descriptive statistics of social media use across a gency levels and multiple waves of the pandemic. We then performed inferential statistics to assess impact of message features on message diffusion. Details on methods can be found at: lhei.org/covid19study.html

The Covid-19 pandemic was selected for this study given its immediate and grave nature as one of the deadliest pandemics in recent history [36], and also to facilitate the analysis in a single crisis domain: public health emergencies. We decided to a nalyze local, state and federal agencies to provide a strong test for the framework in the context of government agencies, and help understand how messages may across agency levels. Twitter was selected given its practicalAPI for data retrieval, and as it is one of the popularplatforms used widely by public health a gencies $[10,27]$.

\subsection{Data collection and annotation}

First, we identified relevant public health agencies for the study. For federal agencies: we identified Twitter accounts for 11 major federal health agencies in the US associated with infection prevention and control; for state agencies: we collected all the Twitter accounts of all 50 state public health agencies. For local agencies: we identified the 50 largest cities in the 50 states, plus DC, and searched for their main local or county public health agency, of which we found a total of 33 Twitter accounts. (See full list of agencies at: lhei.org/covid19study.html).

For the 92 official Twitter accounts identified, we retrieved all tweets (original tweets and replies) from $01 / 01 / 2020$ to $12 / 31 / 2020$. From this dataset, we then retrieved all tweets with a textual reference to: $n c o v$, covid, corona, pandemic, or sars-cov. The earliest covid-19 tweet was on Jan. 11, by the Centers for Disea se Control and Prevention (CDC) main account.

In preparation for the annotation task, we retrieved a random sample of 905 covid-related tweets and replies in a manner proportional to the amount of tweets per agency level in the dataset. The rationale was to sample from the variety of accounts and messages in the population $(\mathrm{N}=51,192$ tweets and replies). Given the detailed manualannotation, the 905 sample is similar to other studies $[9,16]$.

The annotation of messages consisted in a binary coding for the presence of the feature in the text or text-in-image. Three authors trained together and then independently annotated the sample dataset, where ultimately $n=902$. A $20 \%$ sub-sample of tweets were independently double coded to calculate Cohen's kappa statistic of inter-rater reliability (IRR) for each feature [37]. Observed discordance in these results was discussed and finalvalues agreed upon.

\subsection{Analytical Procedures}

RQ1. How reliable are the framework features for analyzing crisis-related social media messages? To address this question, we used Cohen's kappa coefficient, which provides values between 0 and 1 . Levels below .41 are interpreted as weak; levels between .41-.60 are weak to moderate; above .61 moderate to substantial; and between $.81-1$ as strong to almost perfect $[37,38]$.

RQ2. How are message features employed across government agencies throughout the crisis?

We addressed this question by calculating the proportional distribution of message features across agency levels. To provide a long term view, we also visualized Twitter activity over time, across multiple waves of the pandemic. We calculated the 7-day moving averages of: average posts by agency level; total retweets of agency posts by level; and total confirmed covid-19 cases in the US through 2020.

RQ3. How are message features associated with the message diffusion rate (DR)? 
To address this question, we calculated a diffusion rate of message $m\left(D R_{m}\right)$ as the normalized retweet count of message (i.e., post, tweet) $m$ :

$$
D R_{m}=\frac{R T_{m}}{F_{a}}
$$

where $R T_{m}$ is the retweet count of the message, and $F_{a}$ is the follower count of the account that posted the message. Although messages are not only retweeted (i.e., shared, retransmitted) by an account's followers, this measure controls for the account's network size.

For every feature, we computed the mean $D R$ of all messages that contained the feature, and of all messages that did not contain it, and compared these two groups via independent samples Welch's t-test (two-tailed). Although assumptions of normality and equalvariance were not met, and the Wilcoxon-MannWhitney (WMW) test may be best, a number of features have relatively high sample size, indicating Welch's t-test may be preferable [39]. Moreover, the WMW test when applied to our data provided more significant, and untenable, results. We thus report analyses from the Welch's t-test.

\section{Results}

Table 2 shows descriptive statistics. As can be observed, local, state and federal agencies made on average a comparable number of tweets and replies, although state agencies were on average more active. Federal a gency accounts were more popular than state and local a gencies by multiple orders of magnitude

\subsection{Inter-rater reliability for annotation task}

Kappa values above .81 were: question; prevalence; how-to-protect; material resource; political figure; and text-in-image. Kappa values between .61 and .8 were: directive; scientific-information; policy/action; interactive resource; corrective; population group; secondary threat; agency expert/staff; collective frame positive frame; and strong emphasis. Low kappa were: request (.56) and external speaker (.58). Emergent event; metaphor; and personality did not sufficiently appear in this task for reliable ratings to be assessed.

\subsection{Twitter activity and message features across local, state, and federal agencies}

Figure 1 shows the rapid increase in Covid-19 related messages weeks prior to the declaration of a global pandemic by the World Health Organization on March 11. As shown, state agencies are the most active throughout the pandemic. Posting activity subsides in about 3 months, but rises again, mostly for state and
Table 2. Sample (s) and population (p) statistics for tweets related to Covid-19 throughout 2020

\begin{tabular}{|c|c|c|c|c|c|}
\hline & & Local & State & Federal & All \\
\hline \multirow{2}{*}{$\begin{array}{c}\text { Twitter } \\
\text { accounts }\end{array}$} & $s$ & 30 & 45 & 10 & 85 \\
\hline & p & 33 & 48 & 11 & 92 \\
\hline \multirow{2}{*}{$\begin{array}{l}\text { Total tweets } \\
\text { and replies }\end{array}$} & $s$ & 277 & 535 & 90 & 902 \\
\hline & $p$ & 15,699 & 30,408 & 5,022 & 51,129 \\
\hline \multirow{2}{*}{ Total replies } & s & 15 & 53 & 8 & 76 \\
\hline & $\mathbf{p}$ & 1,044 & 3,427 & 641 & 5,112 \\
\hline \multirow{2}{*}{$\begin{array}{l}\text { Mean followers } \\
\text { count per agency } \\
\text { (std. dev.) }\end{array}$} & $s$ & $\begin{array}{c}14,522 \\
(20,440)\end{array}$ & $\begin{array}{c}26,498 \\
(22,924)\end{array}$ & $\begin{array}{c}877,132 \\
(1,122,111)\end{array}$ & $\begin{array}{c}122,346 \\
(460,676)\end{array}$ \\
\hline & p & $\begin{array}{r}13,566 \\
(19,758) \\
\end{array}$ & $\begin{array}{r}25,440 \\
(22,737) \\
\end{array}$ & $\begin{array}{c}860,016 \\
(1,066,041) \\
\end{array}$ & $\begin{array}{c}120,967 \\
(447,558) \\
\end{array}$ \\
\hline \multirow{2}{*}{$\begin{array}{c}\text { Mean retweet } \\
\text { count per tweet } \\
\text { (std. dev.) }\end{array}$} & $s$ & $8.3(28.6)$ & $16.8(29.9)$ & $329.3(1304)$ & $45.2(420)$ \\
\hline & p & $10.3(52.9)$ & $17.6(54.6)$ & $153.6(537.7)$ & $28.2(178.4)$ \\
\hline
\end{tabular}

federal agencies, with the third wave. Figure 1 shows that the initial public response (as retweets of agency posts) is high across a gency levels, but subsides within one month, without large subsequent increases.

Table 3 shows the proportion of local, state, and federal posts that contained each message feature. Local agencies employed the most: question prompt, expressive, how-to-protect, corrective, other language, direct mention, external speaker, strong emphasis, positive frame and text-in-image.

State agencies employed the most representative statements, participatory requests, replies, interactive resources, political figure and collective frame.

Federal agencies employed the most: directive, scientific information, emergent events, action/policy, references to secondary threat and population group, agency expert/staff, personality, and all of the media features except text-in-image. The highest differences are between local and federala gencies.
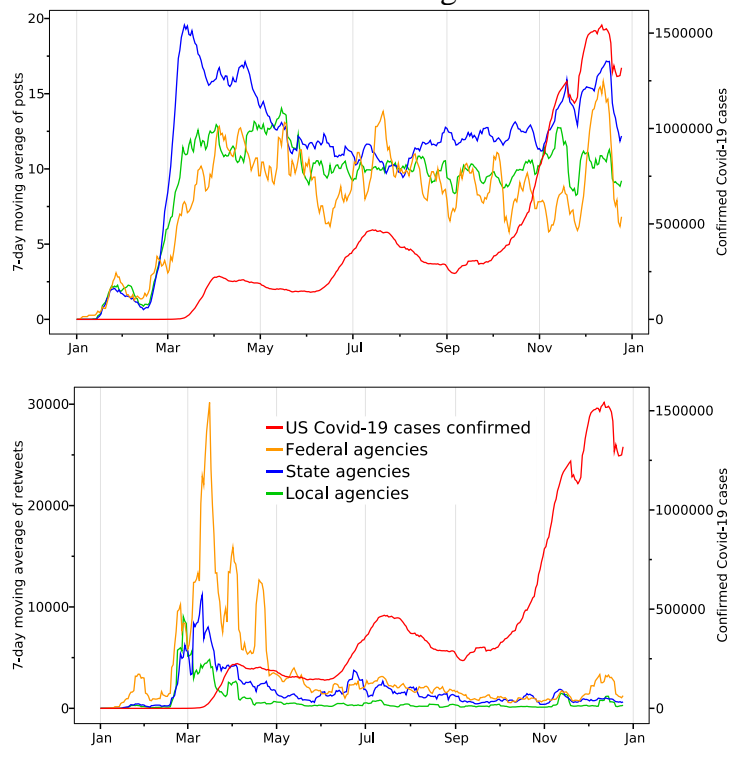

Note: Covid-19 data from Johns Hopkins University at: https://github.com/CSSEGISandData/COVID-19

Figure 1. Average posts per agency, total public retweets and confirmed US Covid-19 cases, 2020 
Table 3. Proportion of local, state and federal posts that contained each feature Proportion of posts with each feature Total Loc $(n=277) \quad$ State $(n=535) \quad$ Fed $(n=90) \quad(n=902)$

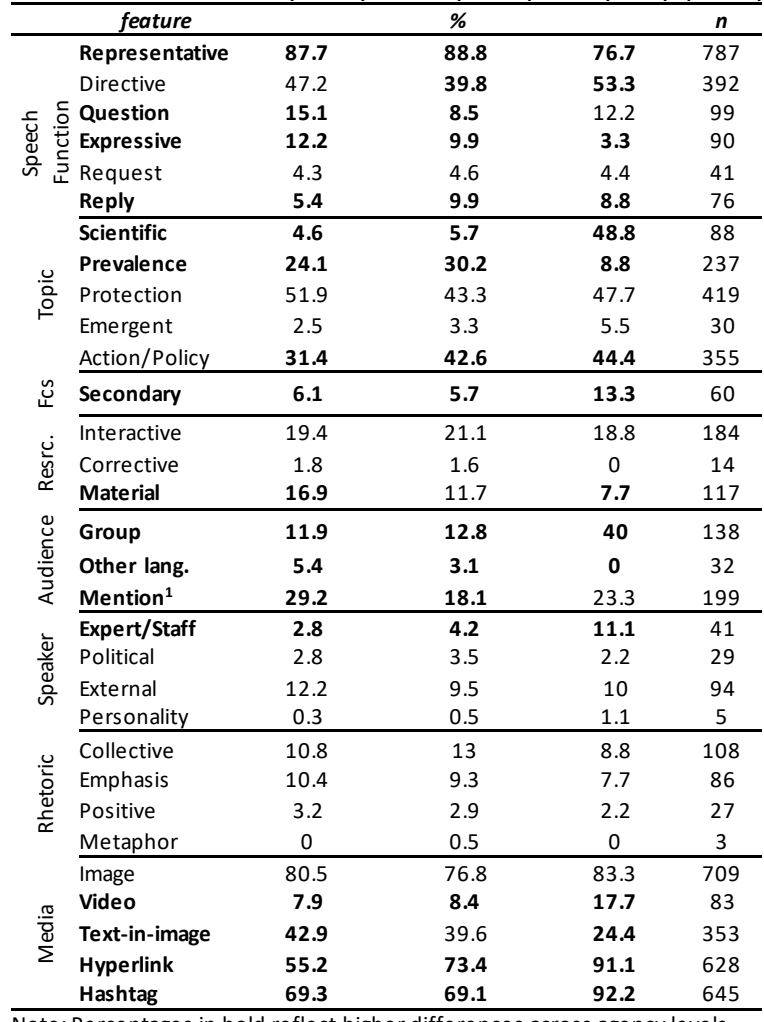

Note: Percentages in bold reflect higher differences across agency levels.

1. Mention calculations do not include direct replies.

Figure 2 shows two illustrative examples of tweets from the sample. The tweet on the left includes an expressive, a collective frame, prevalence information, hashtags, a URL, and text-in-image. It also has an infographic, as it includes statistics and illustrations with how-to-protect instructions. It was retweeted 86 times, or by $.42 \%$ of the agency's follower count.

In contrast, the tweet on the right has a reference to a material resource (i.e. testing site), a weak reference to prevalence ("rapid rise in..."), no statistics, no URL, a simple illustration (the map icon) but without any additional images or text-in-image. The diffusion/retransmission rate of this message was $0.037 \%$, quite below the more common mean diffusion rate (DR) as shown in Table 4.

\subsection{Message features and diffusion rates}

Table 4 presents results of t-tests of differences in mean DR between messages that contained the feature compared to those that did not. Results are read as follows: posts that contained a representative were on a verage retweeted by $.059 \%$ of the follower count of the agency that made the post. This was a statistically

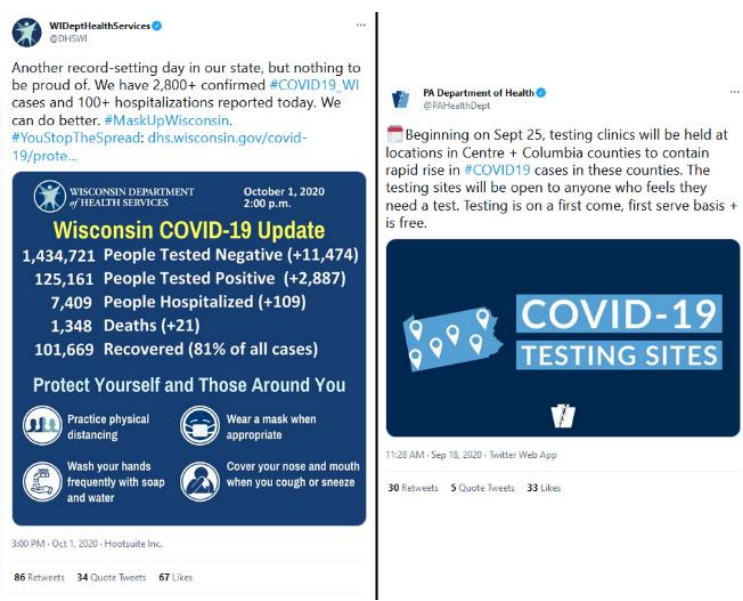

Figure 2. Example posts with varied text and media features (see details at lhei.org/covid19study.html)

significant difference compared to the $.025 \%$ diffusion rate of messages without this feature.

We found that posts containing a representative, prevalence, action/policy, text-in-image tended to be retweeted more frequently. Posts containing a directive, question, scientific, material resource, population group, other language, expert/staff, political, and video were less likely to be retweeted.

Table 4. $t$-tests of differences in mean DR between tweets without (w/o) and with (w/) feature

\begin{tabular}{|c|c|c|c|c|c|c|}
\hline & feature & $\begin{array}{c}w / o \\
\text { feature } \\
\text { mean DR }\end{array}$ & $\begin{array}{c}w / \\
\text { feature } \\
\text { mean DR }\end{array}$ & t-stat & $\begin{array}{c}p- \\
\text { value }\end{array}$ & $\begin{array}{c}\text { sig. } \\
\text { level }\end{array}$ \\
\hline \multirow{6}{*}{ 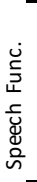 } & Representativ & 0.025 & 0.059 & 5.9 & 0.000 & $* * *$ \\
\hline & Directive & 0.061 & 0.047 & -1.96 & 0.049 & $* *$ \\
\hline & Question & 0.056 & 0.038 & -2.28 & 0.023 & $* *$ \\
\hline & Expressive & 0.053 & 0.065 & 1.03 & 0.304 & n.s. \\
\hline & Request & 0.054 & 0.053 & -0.14 & 0.884 & n.s. \\
\hline & Reply ${ }^{1}$ & 0.055 & 0.008 & - & - & - \\
\hline \multirow{5}{*}{$\frac{0}{\circ}$} & Scientific & 0.056 & 0.037 & -2.89 & 0.004 & $* * *$ \\
\hline & Prevalence & 0.042 & 0.090 & 5.41 & 0.000 & $* * *$ \\
\hline & Protection & 0.058 & 0.050 & -1.16 & 0.243 & n.s. \\
\hline & Emergent & 0.052 & 0.120 & 1.02 & 0.314 & n.s. \\
\hline & Action/Policy & 0.049 & 0.063 & 1.69 & 0.090 & $*$ \\
\hline$\tilde{u}^{-}$ & Secondary & 0.055 & 0.038 & -1.60 & 0.112 & n.s. \\
\hline \multirow{3}{*}{$\begin{array}{l}\stackrel{\dot{U}}{\check{U}} \\
\check{\simeq}\end{array}$} & Interactive & 0.054 & 0.054 & -0.10 & 0.913 & n.s. \\
\hline & Corrective & 0.052 & 0.180 & 0.89 & 0.390 & n.s. \\
\hline & Material & 0.056 & 0.042 & -1.96 & 0.050 & $*$ \\
\hline \multirow{3}{*}{ 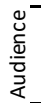 } & Group & 0.059 & 0.025 & -5.28 & 0.000 & $* * *$ \\
\hline & Other lang. & 0.055 & 0.018 & -6.65 & 0.000 & $* * *$ \\
\hline & Mention & 0.055 & 0.051 & -0.42 & 0.668 & n.s. \\
\hline \multirow{4}{*}{ 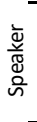 } & Expert/Staff & 0.055 & 0.029 & -3.64 & 0.000 & $* * *$ \\
\hline & Political & 0.055 & 0.023 & -4.16 & 0.000 & $* * *$ \\
\hline & External & 0.055 & 0.045 & -1.21 & 0.226 & n.s. \\
\hline & Personality ${ }^{2}$ & - & - & - & - & - \\
\hline \multirow{4}{*}{$\begin{array}{l}\frac{0}{0} \\
+\frac{1}{0} \\
\frac{1}{x}\end{array}$} & Collective & 0.052 & 0.067 & 1.11 & 0.266 & n.s. \\
\hline & Emphasis & 0.054 & 0.055 & 0.06 & 0.948 & n.s. \\
\hline & Positive & 0.054 & 0.058 & 0.20 & 0.836 & n.s. \\
\hline & Metaphor $^{2}$ & - & - & - & - & - \\
\hline \multirow{5}{*}{$\frac{\frac{\pi}{\pi}}{\frac{d}{2}}$} & Image & 0.056 & 0.054 & -0.19 & 0.847 & n.s. \\
\hline & Video & 0.056 & 0.041 & -1.75 & 0.081 & $*$ \\
\hline & Text-in-image & 0.047 & 0.065 & 2.28 & 0.022 & $* *$ \\
\hline & Hyperlink & 0.055 & 0.054 & -0.05 & 0.952 & n.s. \\
\hline & Hashtag & 0.056 & 0.054 & -0.36 & 0.717 & n.s. \\
\hline
\end{tabular}


The use of most media features were not associated with higher diffusion rates, except for text-in-image, Although not statistically significant, Table 4 shows that messages that referred to an emergent event, had a collective frame or corrective had a relatively higher diffusion rate than messages without those features.

\section{Discussion}

This study was motivated to improve frameworks used to study social media messa ges for crisis and risk communication. We developed a framework focused on textual and media features of a post, integrating categories from the literature, and adding relevant features not previously discussed in similar studies.

We also provide empirical results on analyses of: (1) the inter-rater reliability of the message features from sampled Twitter posts; (2) Twitter activity of US public health agencies during multiple waves of the Covid-19 pandemic in 2020; (3) distribution of various message features across local, state and federal agencies; and (4) analytical tests for the association between message features and message diffusion.

Toward a generalizable framework for social media message analysis. The inter-rater reliability results of our framework indicate the developed framework is promising. In our study most features that required human annotation were above .7 of Cohen's kappa, while a few related to rhetorical tactics and corrective were around the .6 mark. We suggest that the textual and media based nature of this framework helps with the genera lizability of the model across crisis situations, and can help researchers and practitioners give focus to more objective and deeper elements of short text communication.

Previous similar studies often do not report interrater reliability $[12,13]$; have remarkably high levels for rather abstract categories [16, 26]; or use a lexical (keyword) approach without any human annotation $[10,11]$. A similar study found that Facebook messages with "warning", "condolences", or "encouragement" content had Krippendorff's alpha levels between 0.5 to 0.75 [4]. This all suggests that this type of message analysis is difficult, but that our framework is promising and could improve with better definitions of the constructs.

Importance of differentiating communication strategies by government levels. Studies of crisis and risk communication messages on social media usually focus on a single level of government $[4,12,26]$, and when local, state and federal agencies are captured they do not explore how different types of messages may be associated with different types of agencies or levels of government $[10,11]$. Whereas examining local, state and federal agencies functions as a strong test for the reliability and validity of the framework across government agencies, it also enables us to see how agency levels may focus more or less on different types of messages or include different types of message content.

For example, in our study we found that expressives, which mostly refer to expressions of sentiment, are employed almost 4 times more by local agencies than federalagencies. It may be fair to speculate this is due to a closer connection, real or imaged, between local a gencies and their publics, compared to the connection between the public and the federal government.

Scientific information, on the other hand, which refers to more technical information regarding the causes, risks or impacts of the threat was used about 4 times more by federal a gencies compared to state and local agencies. This observation may be due to this specific crisis situation, as a novel coronavirus that surged in China at the end of 2019 and quickly became a global pandemic [36]. In this case, federal agencies such as the National Institutes of Health (NIH) had been researching coronavirus and were paramount in developing the Covid-19 vaccine [40]. We may thus suggest that local and state agencies needed and relied on scientific information from federala gencies.

A number of other interesting, and potentially expected patterns emerged, not all of which can be discussed here. Nevertheless, federalagencies focused more on segmenting messages based on population group, and had ubiquitous use of hyperlinks and hashtags. This may be partially explained by the fact that in federal agencies government communicators need to speak to larger and thus more diverse communities. Local and state agencies were nevertheless more focused on providing prevalence statistics, likely because of the specificities of regional variations of the pandemic progression, and the fact that Covid-19 dashboards and information systems were being largely developed by state agencies [41].

Understanding associations between message features and message diffusion. Our findings have practical implications for government emergency response and public health communication strategies by identifying features associated with message diffusion rates. When the purpose of the social media communication is to improve the diffusion of messages and increase message reach, government agencies can adopt (or avoid) features that are positively (negatively) associated with diffusion rate.

Some notable findings include the significant differences between messages that contained a representative from those that did not, which had been 
previously observed [3]. Representatives are the statements that describe or explain information. It is thus relevant for communicators to recognize to make posts that at least have a single such statement.

Messages from political accounts generally have higher rates of message sharing compared to those from government accounts $[10,11]$, however, in this study when a political figure or agency expert/staff was a subject in an agency message, on average it did not help with message diffusion. This may be because part of the public wants politics out of public health communication, or related to findings that mention of others does not help message diffusion [11, 14]. Government and public health communicators may thus want to be cautioned if making these references.

Correcting misinformation during a pandemic is an important task [30], but previous studies have had mixed results on the impact of this variable on message diffusion [3]. Although results here are not statistically significant, they clearly point in the direction of a positive impact for correctives. This thus suggests that communicators can expect more than a vera ge public engagement when correcting rumors.

In our framework we included distinct types of images that are rarely systematically explored. Images are important in risk communication for attention, recall and comprehension [17], and previous studies all point to the importance of images to increase message diffusion $[4,14,27]$. In general, our results show that the presence of an image did not increase diffusion. However, we were not able not analyze the more specific types of images for this report. Our study does point to the importance of text-in-image as also indicated in a recent study [11]. This suggests that "While style and context can matter, content is key to retransmission potential." [11]. The images are important, but equally or more important is the semantic and textualinformation in the messages.

\section{Limitations and future studies}

To improve on this research, a number of avenues are clearly warranted. First, further formalization of our model is necessary. This study points to the importance in capturing message features that are grounded on the text itself, rather than on assumptions from readers or researchers. But given this objective focus IRR measures should improve. A more literal and text-based approach can also more easily be integrated into an ontology or a formalized semantic network [5, 20]. Automatic classification of message features are possible via machine learning [5].

A second opportunity for future research is to further explore differences across local, state and federal agencies. There are different ways to balance the sample, and our strategy prioritized sampling from most prevalent accounts. Future study can improve with a deeper look into the different needs and tendencies of different agency levels.

Other clear avenues for research include using a higher sample. Although previous studies had been successful with lower samples, given this set of features as used here will likely require a sample of posts at least twice as large (e.g. roughly 2000 posts). Also, developing a more controlled model to test the impact of message features on message diffusion can ultimately validate the findings. Lastly, future research, either from a communication, information systems, or health informatics perspective needs to include and compare messages across social media platforms, which is rarely done in the literature.

\section{References}

[1] CDC, Crisis and Emergency Risk Communication (CERC): Introduction, Centers for Disease Control and Prevention, 2018.

[2] Reynolds, B., and M. Seeger, "Crisis and emergency risk communication as an integrative model", Journal of Health Communication 10(1), 2005, pp. 43-55.

[3] Sutton, J., C.B. Gibson, N.E. Phillips, et al., "A crosshazard analysis of terse message retransmission on Twitter", Proceedings of the National Academy of Sciences 112(48), 2015, pp. 14793-14798.

[4] Ross, B., T. Potthoff, T.A. Majchrzak, N.R.

Chakraborty, M.B. Lazreg, and S. Stieglitz, "The Diffusion of Crisis-Related Communication on Social Media: An Empirical Analysis of Facebook Reactions", Proceedings of the 51st Hawaii International Conference on System Sciences, 2018.

[5] Coche, J., G. Romera Rodriguez, A. Montarnal, A. Tapia, and F. Benaben, "Social media processing in crisis response: An attempt to shift from data to information exploitation.", Proceedings of the 54th Hawaii

International Conference on System Sciences, (2021).

[6] Mergel, I., "Social media institutionalization in the U.S. federal government", Government Information Quarterly 33(1), 2016, pp. 142-148.

[7] Hagen, L., S. Neely, R. Scharf, and T.E. Keller, "Social Media Use for Crisis and Emergency Risk

Communications During the Zika Health Crisis", Digital Government: Research and Practice 1(2), 2020.

[8] Wang, Y., H. Hao, and L.S. Platt, "Examining risk and crisis communications of government agencies and stakeholders during early-stages of COVID-19 on Twitter", Computers in Human Behavior 114, 2021.

[9] Lwin, M.O., J. Lu, A. Sheldenkar, and P.J. Schulz, "Strategic Uses of Facebook in Zika Outbreak Communication: Implications for the Crisis and Emergency Risk Communication Model", International Journal of Environmental Research and Public Health 15(9), 2018. [10] Sutton, J., S.L. Renshaw, and C.T. Butts, "COVID-19: Retransmission of official communications in an emerging pandemic", PLOS ONE 15(9), 2020, pp. e0238491. 
[11] Renshaw, S.L., S. Mai, E. Dubois, J. Sutton, and C.T. Butts, "Cutting Through the Noise: Predictors of Successful Online Message Retransmission in the First 8 Months of the COVID-19 Pandemic", Health Security 19(1), 2021, pp. 31-43.

[12] Subba, R., and T. Bui, "Online Convergence Behavior, Social Media Communications and Crisis Response: An Empirical Study of the 2015 Nepal Earthquake Police Twitter Project", Proceedings of the 50th Hawaii International Conference on System Sciences, (2017). [13] Ehnis, C., and D. Bunker, "Social Media in Disaster Response:Queensland Police Service - Public Engagement During the 2011 Floods", ACIS 2012 Proceedings, (2012). [14] Vos, S.C., J. Sutton, C.B. Gibson, and C.T. Butts, "\#Ebola: Emergency Risk Messages on Social Media", Health Security 18(6), 2020, pp. 461-472.

[15] Imran, M., P. Mitra, and C. Castillo, "Twitter as a Lifeline: Human-annotated Twitter Corpora for NLP of Crisis-related Messages", arXiv:1605.05894 [cs], 2016. [16] Slavik, C.E., C. Buttle, S.L. Sturrock, J.C. Darlington, and N. Yiannakoulias, "Examining Tweet Content and Engagement of Canadian Public Health Agencies and Decision Makers During COVID-19: Mixed Methods Analysis", Journal of Medical Internet Research 23(3), 2021, pp. e24883.

[17] Vos, S.C., and E. Cohen, "Using Pictures in Health and Risk Messages", Oxford Research Encyclopedia of Communication, 2017.

[18] Fairclough, N., Analysing discourse: Textual analysis for social research, Routledge, London, 2003.

[19] van Dijk, T.A., Discourse and knowledge: A sociocognitive approach, Cambridge University Press, Cambridge, U.K., 2014.

[20] Bénaben, F., C. Hanachi, M. Lauras, P. Couget, and V. Chapurlat, "A Metamodel and its Ontology to Guide Crisis Characterization and its Collaborative Management", Proceedings of the 5th International ISCRAM Conference, 2008.

[21] Coombs, W.T., and S.J. Holladay, The Handbook of Crisis Communication., Wiley-Blackwell, 2010.

[22] Auxier, B., and M. Anderson, "Social Media Use in 2021", Pew Research Center: Internet, Science \& Tech, 2021. https://www.pewresearch.org

[23] Vosoughi, S., and D. Roy, "Tweet Acts: A Speech Act Classifier for Twitter", Tenth International AAAI

Conference on Web and Social Media, (2016).

[24] Searle, J., "A Taxonomy of Illocutionary Acts", In Expression and Meaning: Studies in the Theory of Speech Acts. Cambridge University Press, Cambridge, 1979, 1-29. [25] DePaula, N., E. Dincelli, and T.M. Harrison, "Toward a typology of government social media communication: Democratic goals, symbolic acts and self-presentation", Government Information Quarterly 35(1), 2018, pp. 98108.

[26] Malik, A., M.L. Khan, and A. Quan-Haase, "Public health agencies outreach through Instagram during the COVID-19 pandemic: Crisis and Emergency Risk Communication perspective", International Journal of Disaster Risk Reduction 61, 2021.
[27] Vos, S.C., J. Sutton, Y. Yu, et al., "Retweeting Risk Communication: The Role of Threat and Efficacy", Risk Analysis 38(12), 2018, pp. 2580-2598.

[28] Barboza, G.E., L.B. Schiamberg, and L. Pachl, “A spatiotemporal analysis of the impact of COVID-19 on child abuse and neglect in the city of Los Angeles,

California", Child Abuse \& Neglect 116, 2021, pp. 104740. [29] Cullen, W., G. Gulati, and B.D. Kelly, "Mental health in the COVID-19 pandemic", QJM: An International Journal of Medicine 113(5), 2020, pp. 311-312.

[30] Tasnim, S., M.M. Hossain, and H. Mazumder, "Impact of Rumors and Misinformation on COVID-19 in Social Media”, Journal of Preventive Medicine and Public Health 53(3), 2020, pp. 171-174.

[31] Myrick, J.G., "Celebrity-Based Appeals in Health and Risk Messaging”, The Oxford Encyclopedia of Health and Risk Message Design and Processing, 2017.

[32] Hagen, L., T. Keller, S. Neely, N. DePaula, and C. Robert-Cooperman, "Crisis communications in the age of social media: A network analysis of Zika-related tweets", Social Science Computer Review 36(5), 2018, pp. 523-541. [33] Parrott, R., The Oxford Encyclopedia of Health and Risk Message Design and Processing, Oxford University Press, 2017.

[34] DePaula, N., and E. Dincelli, "Information strategies and affective reactions: How citizens interact with government social media content", First Monday 23(4), 2018.

[35] DePaula, N., "\#Supporting the cause: An analysis of how government agencies use Twitter hashtags",

Proceedings of the Association for Information Science and Technology 55(1), 2018, pp. 788-789.

[36] Rosenwald, M., "History's deadliest pandemics, from ancient Rome to modern America", Washington Post, 2021. https://www.washingtonpost.com

[37] Wongpakaran, N., T. Wongpakaran, D. Wedding, and K.L. Gwet, “A comparison of Cohen's Kappa and Gwet's AC1 when calculating inter-rater reliability coefficients: a study conducted with personality disorder samples", $B M C$ medical research methodology 13, 2013, pp. 61 .

[38] Landis, J.R., and G.G. Koch, "The Measurement of Observer Agreement for Categorical Data", Biometrics 33(1), 1977, pp. 159-174.

[39] Fagerland, M.W., "t-tests, non-parametric tests, and large studies - a paradox of statistical practice?", BMC Medical Research Methodology 12(1), 2012, pp. 78. [40] Garnett, C., "Corbett Recounts Quest for Covid Vaccine", NIH Record, 2020.

https://nihrecord.nih.gov/2020/12/11/corbett-recountsquest-covid-vaccine

[41] Dixon, B.E., S.J. Grannis, C. McAndrews, et al., "Leveraging data visualization and a statewide health information exchange to support COVID-19 surveillance and response: Application of public health informatics", Journal of the American Medical Informatics Association: JAMIA, 2021, pp. ocab004. 